http://jmscr.igmpublication.org/home/

ISSN (e)-2347-176x ISSN (p) 2455-0450

crossref DOI: https://dx.doi.org/10.18535/jmscr/v7i7.25

\title{
CBNAAT: Comparision of Diagnositc Yield in Sputum and Bal Fluid
}

\section{Authors \\ Dr Santosh Kumar, MD¹, Dr Gajendra Vikram Singh ${ }^{2} *$, MD, Dr Avinash Kumar ${ }^{3}$, Dr Rishabh Goel ${ }^{4}$, Dr Parul Mittal ${ }^{5}$}

${ }^{1}$ Proff. and Head of the department, Deptt. of Tuberculosis and Respiratory Diseases, S.N. Medical College, Agra, (UP) India

${ }^{2}$ Associate Proff., Deptt. of Tuberculosis and Respiratory Diseases, S.N. Medical College, Agra, (UP) India

${ }^{3,5}$ JRIII, Deptt. of Tuberculosis and Respiratory Diseases, S.N. Medical College, Agra, (UP) India

${ }^{4}$ Senior Resident Deptt. of Tuberculosis and Respiratory Diseases, S.N. Medical College, Agra, (UP) India

*Corresponding Author

\section{Dr Gajendra Vikram Singh}

Md, Associate Proff., Deptt. of Tuberculosis and Respiratory Diseases, S.N. Medical College, Agra, (UP) India

\begin{abstract}
Aims and Objectives: To compare the diagnostic yield of CBNAAT method for BAL fluid and sputum for detection of mycobacterium to diagnose pulmonary tuberculosis.

Material and Methods: 129 sputum and BAL specimens from 129 patients were studied which included 65 specimens from sputum culture positive TB patients and 64 specimens from sputum culture negative patients. Patients with Sputum culture negative for MTB were considered as control.

Observation: It was observed that out of 65 culture positive cases of pulmonary tuberculosis, 43 were sputum CBNAAT (+)ve and 54 were CBNAAT (+)ve in BAL. Sensitivity and specificity of CBNAAT for sputum was $66.15 \%$ and $100 \%$ respectively. Sensitivity and specificity of CBNAAT for BAL was $83.07 \%$ and $100 \%$ respectively.

Conclusion: our study suggests that BAL-CBNAAT shows additional advantage of confirmatory diagnosis of the disease even if sputum CBNAAT is negative.
\end{abstract}

\section{Introduction}

Tuberculosis is caused by bacteria (mycobacterium tuberculosis) that most often affects lungs ${ }^{1}$. Tuberculosis occurs in every part of the world. Tuberculosis is one of the top ten causes of death worldwide. In 2017, 10 million people fell ill with $\mathrm{TB}$, and 1.6 million died from the disease (including 0.3 million among people with HIV). With the pandemic of AIDS and emergence of drug resistant strains of mycobacterium tuberculosis, pulmonary tuberculosis has now become a major health problem to the world community ${ }^{2}$.

TB spreads from person to person through air. When person with pulmonary TB, coughs, sneeze or spits they propel the bacteria and inhalation of these bacteria can cause TB infection ${ }^{1}$.

Early and rapid diagnosis and treatment of the case is the most important step in reducing the TB incidence ${ }^{3}$. 
Global incidence is falling at about $2 \%$ per year. This needs to accelerate to a $4-5 \%$ annual decline to reach the 2020 milestones of the End TB strategy ${ }^{4}$.

Lab diagnosis of TB is based on traditional method of smear microscopy by Ziel-neelsen staining and on sputum culture of Mycobacterium Tuberculosis. Sputum microscopy is rapid and inexpensive but bacterial concentration required is 10,000 Acid Fast Bacillii per ml or greater. It also lacks specificity and cannot distinguish various strains of mycobacterium. Culture of Mycobacterium tuberculosis is more sensitive and 10-100 viable bacilli are required but it takes long period to produce results.

The detection of Mycobacterium Tuberculosis by Cartridge Based Nucleic Acid Amplification Test (CBNAAT) has been found to be useful in diagnosis of pulmonary tuberculosis.

The CBNAAT is one of these newer methods that simultaneously identifies Mycobacterium tuberculosis and detects rifampicin resistance ${ }^{5}$.

In our study we have compared the yield of CBNAAT method for BAL fluid and sputum for detection of mycobacterium to diagnose tuberculosis.

\section{Material and Method}

This was a hospital based comparative study, conducted in TB and chest Department of SN Medical College Agra

We have investigated presumptive pulmonary TB cases. Detailed clinical history was taken and chest x-ray \& sputum smear microscopy after ZN staining was done.

All cases were sent to Intermediate reference laboratory National JALMA Institute for Leprosy and Other Mycobacterial Diseases for sputum culture for MTB. 129 sputum and BAL specimens

\begin{tabular}{|l|c|c|c|c|c|}
\hline \multirow{2}{*}{ Group } & \multirow{2}{*}{ Total } & \multicolumn{2}{|c|}{ Sputum for CBNAAT } & \multicolumn{2}{c|}{ BAL for CBNAAT } \\
\cline { 3 - 6 } & & $(+) \mathrm{ve}$ & $(-) \mathrm{ve}$ & $(+) \mathrm{ve}$ & $(-) \mathrm{ve}$ \\
\hline Pul. TB & 65 & 43 & 22 & 54 & 11 \\
\hline Sputum Culture Negative cases & 64 & 0 & 64 & 0 & 64 \\
\hline
\end{tabular}

It was observed that out of out of 65 culture positive cases of pulmonary tuberculosis, 43 were from 129 patients were studied which included 65 specimens from sputum culture positive TB patients and 64 specimens from sputum culture negative patients. Patients with Sputum culture negative for MTB were considered as control.

Sputum samples were collected according to RNTCP guidelines and samples sent to Intermediate reference laboratory, State Tuberculosis Training \& Demonstration Centre Agra for CBNAAT.

Bronchoalveolar lavage was taken in our bronchoscopy suit in the department of Respiratory Medicine, SN Medical College, Agra. BAL fluid sent to Intermediate reference laboratory State Tuberculosis Training \& Demonstration Centre Agra for CBNAAT.

CBNAAT: Cartridge-based nucleic acid amplification test (CBNAAT) is a recently introduced polymerase chain reaction (PCR) based method for detection of Tuberculosis.It also detects rifampicin resistance as it targets the rpoB gene of mycobacteria. CBNAAT is a Mycobacterium tuberculosis-specific automated, cartridgebased nucleic acid amplification assay, having fully integrated and automated amplification and detection using real-time PCR, providing results within 100 minutes. It is a highly specific test as it uses 3 specific primers and 5 unique molecular probes to target the rpoB gene of $\mathrm{M}$. tuberculosis, which is the critical gene associated with rifampicin resistance . $^{6}$

\section{Observation}

The present study was conducted on 129 patients of presumptive tuberculosis, out of which 65 patients Sputum Culture positive cases and 64 patients were Sputum Culture Negative cases, attended department of TB and chest diseases, SN Medical College, Agra. 


\begin{tabular}{|l|c|c|c|}
\hline Sputum for CBNAAT & No. of cases & Control & Total \\
\hline Positive & 43 (True positive) & 0 (False positive) & 43 \\
\hline Negative & 22 (False negative) & 64 (True negative) & 86 \\
\hline Total & 65 & 64 & 129 \\
\hline
\end{tabular}

\begin{tabular}{|c|c|c|}
\hline $\begin{array}{l}\text { Sensitivity } \\
\text { Specificity }\end{array}$ & $\begin{array}{l}=43 / 65=66.15 \% \\
=65 / 65=100.0 \%\end{array}$ & $\begin{array}{l}\text { (+)vePredictive Value } \\
\text { (-)ve predictive Value }\end{array}$ \\
\hline
\end{tabular}

\begin{tabular}{|l|c|c|c|}
\hline BAL for CBNAAT & No. of cases & Control & Total \\
\hline Positive & 54 (True positive) & 0 (False positive) & 54 \\
\hline Negative & 11 (False negative) & 64 (True negative) & 75 \\
\hline Total & 65 & 64 & 129 \\
\hline
\end{tabular}

Sensitivity $=54 / 65=83.07 \%$

Specificity $=64 / 64=100.0 \%$

(+)vePredictive Value

(-)ve predictive Value

$$
\begin{aligned}
& =54 / 54=100.0 \% \\
& =64 / 75=85.33 \%
\end{aligned}
$$

\section{Discussion}

CBNAAT should not be thought as a substitute for culture and SM. However, it can act as an adjuvant of traditional tests and clinical data for confirming TB. It cannot be used for monitoring the therapeutic response as it can produce falsepositive results in presence of non-viable TB bacteria; though identification of $\mathrm{M}$. tuberculosis out of Non-Tubercular Mycobacterium (NTM) is possible by it ${ }^{7}$. Thus, CBNAAT is helpful diagnostic test towards AFB smear-positive patients for rapidly detection of pulmonary $\mathrm{TB}$ and getting it differentiated from $\mathrm{NTM}^{8}$.

A study conducted by Avashia $\mathrm{S}$ et al., reported $47.2 \%$ gain in respect of sputum positivity among smear negative TB cases ${ }^{9}$. The results of the present study have concurrence with this where $66.15 \%$ yield for sputum CBNAAT and for BALCBNAAT it was $83.07 \%$. This $16.92 \%$ increase in the yield arising out of BAL-CBNAAT over and above the yield of sputum CBNAAT was also revealed to be statistically significant. Here, the yield of $16.92 \%$ sputum positive patients would be taken as a major gain from the epidemiological point of view so far as the transmission of TB concerns.

A Cochrane systematic review done in 2013 showed high accuracy of CBNAAT compared to culture. It showed about $88 \%$ sensitivity and $98 \%$ specificity for pulmonary TB in adults. Among smear-negative TB patients, Xpert had a sensitivity of $67 \%{ }^{10}$. Ioannidis $\mathrm{P}$ et al., reported that GeneXpert MTB/RIF assay has positive predictive values for pulmonary and extrapulmonary samples $93.5 \%$ and $50 \%$, whereas negative predictive values for those are $91.7 \%$ and $100 \%$, respectively. In case of microscopically negative specimens, the figures are $79 \%$ and $95.6 \%{ }^{11}$. In our study sensitivity and specificity of sputum for CBNAAT to detect AFB in smear negative cases is $66.15 \%$ and $100.0 \%$.

\section{Conclusion}

Our study reaffirmed the usefulness of the CBNAAT over and above the traditional smear microscopy for a significantly higher yield. It leads to early detection and treatment of TB for stopping the transmission of the disease in the community. BAL-CBNAAT shows additional advantage of confirmatory diagnosis of the disease even if sputum CBNAAT is negative. Simplicity, sensitivity, speed and automation of CBNAAT makes this technique a very attractive tool for diagnosis of Mycobacterium tuberculosis from smear negative cases of TB suspects. With the added advantage of detection of multi-drug resistant cases, it seems to be another mile stone in 'End TB' strategy.

\section{References}

1. WHO. October 2015. Archived from the original on 23 August 2012. Retrieved 11 February 2016. 
2. "Global tuberculosis report". World Health Organization. Retrieved 2017-1109.

3. "End TB Strategy" https://www.who.int/tb/strategy/end-tb/en/

4. https://www.who.int/news-room/factsheets/detail/tuberculosis 18th September 2018.

5. World Health Organization: WHO report 2010: global tuberculosis control. Geneva, Switzerland: WHO; 2010.

6. Rachow A, Zumla A, Heinrich $\mathrm{N}$ et al. Rapid and accurate detection of Mycobacterium tuberculosis in sputum samples by Cepheid Xpert MTB/RIF assay - a clinical validation study. PLoS One 2011; 6: e20458.

7. Centers for Disease Control and Prevention (CDC). Updated guidelines for the use of nucleic acid amplification tests in the diagnosis of tuberculosis. MMWR Morb Mortal Wkly Rep. 2009;58:7-10.

8. Kwon YS, Koh WJ. Diagnosis of pulmonary tuberculosis and nontuberculous mycobacterial lung disease in Korea. Tuberc Respir Dis. 2014;77:1-5.

9. Avashia S, Choubey S, Mishra S, kharate A. To study the usefulness of CBNAAT (cartridge based nuclear acid amplification test) in BAL (bronchoalveolar lavage) samples in the diagnosis of smearnegative/non sputum producing patients with suspected tuberculosis. J Evolution Med Dent Sci. 2016;5(1):55-59.

10. Steingart KR, Sohn H, Schiller I, Kloda LA, Boehme CC, Pai M, et al. Cochrane Library 2013 http://doi.wiley.com/10.1002/14651858.C D009593.pub2

11. Ioannidis P, Papaventsis D, Karabela S, Nikolaou S, Panagi M, Raftopoulou E, et al. Cepheid GeneXpert MTB/RIF assay for mycobacterium tuberculosis detection and rifampin resistance identification in patients with substantial clinical indications of tuberculosis and smearnegative microscopy results. Journal of Clinical Microbiology. 2011;49(8):306870. 